\title{
pharma-kritik
}

\section{Jahrgang 31}

Nummer 16/2009

Medikamentös induzierte Sehstörungen

Verschiedene Medikamente können Ametropien und Akkomodationsstörungen verursachen. Häufig sind dabei Medikamente mit anticholinergischen Eigenschaften im Spiel. Bei anderen Substanzen ist der Mechanismus der Sehstörung nicht immer klar.

Entzündungshemmer für Herzkranke

Die nicht-steroidalen Entzündungshemmer können nicht nur gastro-intestinale, sondern auch kardiovaskuläre und renale Probleme verursachen. Bei Personen mit Herz-Kreislauferkrankungen sollten sie wenn überhaupt notwendig - in möglichst kleiner Dosis und nur für kurze Zeit eingesetzt werden.

Flugreisen und Venenthrombosen

Langanhaltendes Stillsitzen kann zu einer thromboembolischen Erkrankung führen. Flugreisen, die nicht länger als 4 Stunden dauern, erhöhen das Thromboserisiko aber nicht nennenswert. Personen mit einem erhöhten Thromboserisiko sollten vor Langstreckenflügen antikoaguliert werden.

\section{Mini-Übersicht}

\section{Medikamentös induzierte Sehstörungen}

Medikamente können zu Ametropien - Myopie und Hyperopie - und zu Akkomodationsstörungen führen und auch auf andere Weise Sehstörungen verursachen. In der Ausgabe vom Januar 2010 von «La Revue Prescrire» findet sich dazu ein Übersichtsartikel. ${ }^{1}$

\section{Merkmale medikamentöser Störungen}

Medikamentös verursachte Ametropien und Akkomodationsstörungen sind meistens bilateral, treten plötzlich auf, können aber auch rasch wieder verschwinden. Subjektiv klagen die Betroffenen meistens darüber, «unscharf» zu sehen. Wie sich die Störung auswirkt, ist nicht nur vom verursachenden Medikament, sondern auch von der vorbestehenden Sehschärfe und weiteren individuellen Faktoren abhängig. Tritt plötzlich und unerklärt eine Sehstörung auf, sollte man in jedem Fall überprüfen, ob die betroffene Person erst gerade mit der Einnahme eines neuen Medikamentes begonnen hat. Sofern eine medikamentöse Ursache vermutet wird und das entsprechende Medikament abgesetzt werden kann, bestätigt sich die Diagnose von selbst, wenn die Sehstörung nach Absetzen bald verschwindet.

\section{Medikamente mit anticholinergischer Wirkung}

Atropin ist der Prototyp der Muscarinrezeptor-Antagonisten, die (unter anderem) zu einer Akkomodationsstörung und Mydriase führen. So ergibt sich eine Pseudopresbyopie, die sich vor allem bei jungen Leuten störend auswirkt. Bei Personen mit einem engen iridokornealen Winkel kann die Mydriase zu einem Engwinkelglaukom führen.

Direkt am Auge werden solche Medikamente in erster Linie angewendet, um eine Zykloplegie (Lähmung des Ziliarmuskels) zu erreichen. Neben Atropin und Scopolamin werden dazu Cyclopentolat $\left(\right.$ Cyclogyl ${ }^{\circledR}$ ) und Tropicamid (Mydriaticum Dispersa, Tropicamide Favre) verwendet. Tropicamid weist eine relativ kurze Wirkungsdauer von wenigen Stunden auf. Die anticholinergischen Wirkungen der anderen Präparate können (nicht nur am Auge, sondern eventuell auch systemisch) vereinzelt viele Tage lang andauern.

Mehrere Medikamente, die zur Behandlung gastro-intestinaler und urologischer Probleme eingesetzt werden, haben anticholinergische Eigenschaften. Dazu gehören z.B. Spasmolytika wie Metixen (in Spasmo-Canulase ${ }^{\circledR}$ ) und Scopolaminbutylbromid (Buscopan ${ }^{\circledR}$ ) sowie Medikamente, die bei Reizblase-Beschwerden verschrieben werden (Tolterodin $=$ Detrusitol ${ }^{\circledR}$ und viele andere).

In der Neurologie und Psychiatrie werden ebenfalls Medikamente verwendet, die mehr oder weniger ausgeprägte anticholinergische Eigenschaften haben. Beispiele sind die trizyklischen Antidepressiva (weniger auch die selektiven SerotoninWiederaufnahmehemmer), viele Neuroleptika, mehrere Parkinson-Therapeutika (z.B. Biperiden $=$ Akineton $^{\circledR}$ ). Auch Amantadin (Symmetrel ${ }^{\circledR}$ u.a.) und Memantin (Axura ${ }^{\circledR}$, Ebi$\mathrm{xa}^{\circledR}$ ) haben anticholinergische Wirkung.

Anticholinergische Effekte an den Augen können selbstverständlich auch unter Ipratropium (Atrovent ${ }^{\circledR}$ u.a.) und Tiotropium (Spiriva ${ }^{\circledR}$ ) beobachtet werden. Dies gilt auch für die meisten älteren $\mathrm{H}_{1}$-Antihistaminika wie z.B. Hydroxyzin $\left(\operatorname{Atarax}^{\circledR}\right)$. 


\section{Cholinergisch wirkende Medikamente}

Medikamente, die zu einer Acetylcholin-Wirkung auf die parasympathischen Nervenfasern führen, wirken sich ebenfalls auf die Augen aus: sie erzeugen einen Akkomodations-Spasmus und eine Miose. Es handelt sich vorwiegend um MuscarinRezeptoragonisten oder Cholinesterasehemmer.

Direkt am Auge werden Acetylcholin (Miochol ${ }^{\circledR}$ E) und Carbachol (Miostat ${ }^{\circledR}$ ) bei Augenoperationen sowie Pilocarpin $\left(\right.$ Spersacarpine $^{\circledR}$ ) bei Glaukom verwendet, letzteres allerdings wegen der Sehstörungen - nur ausnahmsweise.

Systemisch werden Cholinesterasehemmer bei Myasthenien und intestinaler Atonie verschrieben: die entsprechenden Substanzen heissen Neostigmin (Prostigmin ${ }^{\circledR}$ ) und Pyridostigmin (Mestinon ${ }^{\circledR}$ ). Drei Cholinesterasehemmer werden zur Behandlung der Alzheimer-Demenz eingesetzt: Donepezil (Aricept ${ }^{\circledR}$ ), Galantamin (Reminyl ${ }^{\circledR}$ ) und Rivastigmin $\left(\right.$ Exelon $\left.^{\circledR}\right)$. Auch das Zytostatikum Irinotecan (Campto ${ }^{\circledR}$ u.a.) hat Cholinesterasehemmer-Eigenschaften.

Varenicilin (Champix ${ }^{\circledR}$ ), ein partieller NikotinrezeptorAgonist, der zur adjuvanten Therapie beim Tabakentzug empfohlen wird, kann ebenfalls verschiedene Augensymptome verursachen.

\section{Andere Medikamente}

Es gibt eine Reihe von weiteren Medikamenten, die Sehstörungen (Myopie, Akkomodationsstörungen) hervorrufen können, wobei man sich über den genauen Mechanismus oft nicht im Klaren ist.

Topiramat (Topamax ${ }^{\circledR}$ u.a.) und verschiedene Diuretika können zu Veränderungen des Flüssigkeitsgehalts okulärer Strukturen führen, was eine Verengung der vorderen Augenkammer und ein Ödem des Ziliarkörpers verursacht. Damit wird der Abfluss des Kammerwassers behindert und der Augeninnendruck kann ansteigen.

Ähnliche Probleme wurden auch unter Karboanhydrasehemmern in oraler Form (Acetazolamid $=$ Diamox $^{\circledR}$, Glaupax ${ }^{\circledR}$ ) oder als Augentropfen $\left(\right.$ Brinzolamid $=$ Azopt $^{\circledR}$, Dorzolamid $=$ Trusopt $^{\circledR}$ u.a.) beobachtet.

Sulfonamide und besonders die Kombination Cotrimoxazol (Bactrim $^{\circledR}$ u.a.) können eine Myopie verursachen, die möglicherweise allergisch bedingt ist.

Chloroquin (Nivaquine ${ }^{\circledR}$ u.a.) und Hydroxychloroquin (Plaquenil $^{\circledR}$ ) können einerseits zu schwerwiegenden Augenveränderungen (Keratopathie, Retinopathie), anderseits vereinzelt auch zu verschwommenem Sehen ohne klare Ursache führen.

Nicht nur unter Topiramat, sondern auch unter anderen Antiepileptika (Carbamazepin $=$ Tegretol $^{\circledR}$ u.a.; Ethosuximid = Pethinimid $^{\circledR}$ ) kommen Sehstörungen vor.

Lithiumsalze verursachen besonders bei jungen Leuten Akkomodationsstörungen.

Einzelfälle von Sehstörungen wurden ferner unter Metronidazol (Flagyl ${ }^{\circledR}$ u.a.), Cyclophosphamid (Endoxan ${ }^{\circledR}$ ), antilymphozytären Immunglobulinen, Flecainid (Tambocor ${ }^{\mathbb{B}}$ ), Glibenclamid (Daonil ${ }^{\circledR}$ u.a.), Isotretinoin (Roaccutan ${ }^{\circledR}$ u.a.), Chinin und Isosorbiddinitrat (Isoket ${ }^{\circledR}$ u.a.) beobachtet.

\section{Zusammengefasst von E. Gysling}

\section{Literatur}

1 Anon. Rev Prescr 2010; 30: 24-8

\section{Mini-Übersicht}

\section{Entzündungshemmer für Herzkranke}

Die nicht-steroidalen Entzündungshemmer («Antirheumatika») sind in Verruf geraten, weil sie nicht nur gastrointestinale, sondern auch kardiovaskuläre und renale Probleme verursachen können. Deshalb werden heute oft Schmerzmittel ohne entzündungshemmende Eigenschaften verschrieben, obwohl diese in vielen Fällen den Antirheumatika unterlegen sind. ${ }^{1}$

\section{Eine aktuelle Übersicht zum Thema}

Im britischen «Drug and Therapeutics Bulletin» ist im März 2010 eine Übersicht erschienen zur Frage, ob und wie bei Personen mit Herz-Kreislauf-Erkrankungen Antirheumatika eingesetzt werden können. ${ }^{2}$ Einleitend wird rekapituliert, dass nicht-steroidale Entzündungshemmer ZyklooxygenaseHemmern (COX-Hemmern) entsprechen und dass angenommen werden kann, dass die ungünstigen Wirkungen auf Magen und Darm durch die Hemmung von COX-1 verursacht sind.

Im Kreislaufsystem existiert idealerweise ein Gleichgewicht zwischen der thrombosefördernden Wirkung von Thromboxan-A2 (das mithilfe von COX-1 gebildet wird) und der antithrombotischen Wirkung von Prostacyclin (das mithilfe von COX-2 gebildet wird). Acetylsalicylsäure hemmt COX1 in den Blutplättchen irreversibel - für die Lebensdauer der betroffenen Plättchen -, aber auch COX-2 in den Endothelzellen, in denen Prostacyclin jederzeit neu gebildet werden kann. Damit verschiebt die Acetylsalicylsäure das Gleichgewicht in die «antithrombotische» Richtung. Dagegen ist die Wirkung der Antirheumatika auf die COX-1 der Blutplättchen reversibel und betrifft damit stärker die COX-2, was zu einer thrombosefördernden Wirkung führen kann.

\section{Auswirkungen auf Herz und Kreislauf}

Rofecoxib $\left(\right.$ Vioxx $\left.^{\circledR}\right)$ wurde bekanntlich zurückgezogen, weil es im Vergleich mit Placebo häufiger zu Herzinfarkten und Schlaganfällen führte. Die heute zur Verfügung stehenden Daten betreffen in erster Linie Celecoxib (Celebrex ${ }^{\circledR}$ ), Diclofenac (Voltaren ${ }^{\circledR}$ u.a.), Ibuprofen (Brufen ${ }^{\circledR}$ u.a.) und Naproxen (Proxen ${ }^{\circledR}$ u.a.). Zu anderen Antirheumatika ist nur wenig bekannt.

Gemäss einer Meta-Analyse von 138 kontrollierten Studien, in denen COX-2-Hemmer mit Placebo oder nicht-selektiven Antirheumatika verglichen wurden, sind insbesondere Herzinfarkte unter COX-2-Hemmern signifikant häufiger als in den Vergleichsgruppen. Kardiovaskuläre Ereignisse kommen jedoch ähnlich häufig auch unter anderen Antirheumatika vor. Sie sind z.B. unter Diclofenac ebenfalls signifikant häufiger als unter Placebo. ${ }^{3}$ Celecoxib verursacht gemäss einer Meta-Analyse nicht mehr kardiovaskuläre Komplikationen als andere Antirheumatika; ${ }^{4}$ eine andere MetaAnalyse lässt jedoch für Celecoxib auf ein erhöhtes Herz- 
infarkt-Risiko schliessen. ${ }^{5}$ Dass Etoricoxib (Arcoxia ${ }^{\circledR}$ ) $\mathrm{zu}$ einem Blutdruckanstieg führen kann und mehr kardiovaskuläre Probleme als Naproxen verursacht, haben wir vor kurzem berichtet. $^{6}$

Meta-Analysen, die neben randomisierten Studien noch weitere Daten verwerten, ergeben ungefähr dieselben Schlussfolgerungen. Obwohl die Datenbasis schmal ist, lässt sich annehmen, dass Naproxen - im Gegensatz zu Diclofenac und Ibuprofen - eine etwas bessere kardiovaskuläre Verträglichkeit hat als die COX-2-Hemmer. ${ }^{7}$

\section{Anwendung bei Herz-Kreislauf-Kranken}

Die Anwendung von Antirheumatika ist spezifisch bei Personen mit Herz-Kreislauf-Erkrankungen nur wenig untersucht worden. In einer Doppelblindstudie fand sich bei Kranken mit Arthrose und Hypertonie ein Anstieg oder eine Destabilisierung des Blutdrucks sowohl unter Rofecoxib als auch unter Celecoxib und Naproxen. ${ }^{8}$

In einer grossen dänischen Beobachtungsstudie zeigte sich bei Personen nach einem Herzinfarkt ein erhöhtes Mortalitätsrisiko, wenn sie einen COX-2-Hemmer, Diclofenac (>100 mg/Tag) oder Ibuprofen (>1200 mg/Tag) einnahmen. ${ }^{9}$ Gemäss verschiedenen Studien müssen Kranke mit einer Herzinsuffizienz häufiger wegen einer Zunahme der Herzinsuffizienz behandelt oder hospitalisiert werden, wenn sie Antirheumatika erhalten. ${ }^{10,11}$

\section{Schlussfolgerungen}

COX-2-Hemmer und nicht-selektive Antirheumatika erhöhen das Risiko kardiovaskulärer Ereignisse in variablem Ausmass. Wahrscheinlich wirken sie sich besonders dann ungünstig aus, wenn sie längere Zeit in höheren Dosen verabreicht werden.

Lässt sich die Verabreichung von Antirheumatika nicht vermeiden, so soll deshalb auf eine möglichst niedrige Dosis und eine kurze Verabreichungsdauer geachtet werden. Die aktuell vorliegenden Daten lassen vermuten, dass unter den gebräuchlichen Antirheumatika Naproxen das kleinste, Diclofenac das grösste kardiovaskuläre Risiko darstellt. Problematisch ist die Antirheumatika-Gabe insbesondere bei Personen mit einer Herzinsuffizienz-Anamnese. Das Magenrisiko darf nicht vernachlässigt werden und erfordert oft die Verabreichung einer magenschützenden Therapie.

\section{Zusammengefasst von E. Gysling}

\section{Literatur}

1 Towheed TE et al. Cochrane Database Syst Rev 2006; (1): CD004257

2 Anon. Drug Ther Bull 2010; 48: 26-9

3 Kearny PM et al. BMJ 2006; 332: 1302-9

4 White WB et al. Am J Cardiol 2007; 99: 91-8

5 Chen YF et al. Health Technol Assess 2008; 12: 1-278

6 Gysling E. pharma-kritik 2009; 31: 22-4

7 http://compare-analgesics.notlong.com/

8 Sowers JR et al. Arch Intern Med 2005; 165: 161-9

9 Gislason GH et al. Ciculation 2006; 113: 2906-12

10 Feenstra J et al. Arch Intern Med 2002; 162: 265-70

11 Mamdani M et al. Lancet 2004; 363: 1751-6

\section{Mini-Übersicht}

\section{Flugreisen und Venen- thrombosen}

Langanhaltendes Stillsitzen, wie es besonders bei Flugreisen vorkommt, kann das Risiko einer tiefen Venenthrombose und einer Lungenembolie erhöhen. Die Zeitschrift «Australian Prescriber» enthält in ihrem Heft vom Dezember 2009 zu diesem Thema eine Übersicht, ${ }^{1}$ die hier kurz zusammengefasst wird.

\section{Risiko für Gesunde}

Das Risiko einer Thrombose oder einer Lungenembolie hängt von folgenden Umständen ab: Dauer des Fluges lang andauernde Immobilisierung - Behinderung des venösen Rückflusses aus den Beinvenen - Verminderung des Blutflusses als Folge der Dehydratation während des Fluges. Reisende haben daneben eventuell individuelle Risikofaktoren wie z.B. die Verwendung oraler Kontrazeptiva oder eine Thrombose in der Vorgeschichte.

In einer grossen Studie wurde gezielt danach gesucht, ob einzelne Merkmale ein signifikant erhöhtes Risiko bedeuten. Flugreisen, die weniger als 4 Stunden (entsprechend etwa $4000 \mathrm{~km}$ ) dauern, erhöhen das Risiko einer Thromboembolie praktisch nicht. Eine längere Flugdauer ist mit zunehmendem Thromboembolie-Risiko verbunden. Aber auch bei länger dauernden Flügen wird nur bei 1 von ungefähr 4500 gesunden Passagieren eine Thrombose (oder Embolie) manifest. Meistens ist eine solche Komplikation schon sofort und kurz nach dem Flug symptomatisch. 2 bis 4 Wochen nach einem Langstreckenflug entspricht das Risiko wieder demjenigen von Personen, die nicht geflogen sind. ${ }^{2}$

Auch gesunde Menschen können Risikofaktoren aufweisen, die für ein thromboembolisches Ereignis prädisponieren. Allgemein haben Frauen ein etwas höheres Risiko als Männer. Sitzen Gesunde während 4 Stunden unverändert in einem Flugzeugsitz, so lassen sich bereits Beinödeme beobachten. Bei übergewichtigen und relativ kleinen Personen ist damit zu rechnen, dass der venöse Rückfluss im Sitzen stärker als bei anderen Leuten beeinträchtigt ist. Da das Thromboembolie-Risiko vom Stillsitzen abhängt, ist es wohl nicht nur bei Flugreisen, sondern auch bei langen Busreisen u.ä. erhöht.

Bei gesunden und kranken Menschen bedeutsame Faktoren, die das Thromboembolie-Risiko bei Langstreckenflügen erhöhen, sind in der Tabelle 1 zusammengestellt.

\section{Die Pille}

Orale Kontrazeptiva erhöhen das Thromboembolie-Risiko generell. Präparate der neueren «Generationen» sind in dieser Hinsicht eher problematischer als die älteren Levonorgestrel-haltigen Präparate. ${ }^{3}$ 
Tabelle 1: Was erhöht das Thromboserisiko bei einem Langstreckenflug?

\author{
Mässig erhöhtes Risiko \\ - Einnahme oraler Kontrazeptiva \\ - Übergewicht \\ - Geringe Körpergrösse \\ - Genetisch bedingte Thrombophilie \\ - Varizen \\ Deutlich erhöhtes Risiko \\ - Thrombose-Anamnese \\ - Kurz zurückliegende chirurgische Eingriffe \\ - Herzinsuffizienz \\ - Aktive Krebserkrankungen \\ - Kombination von Faktoren mit mässigem Risiko \\ - Sehr lange Flugreisen (>10’000 km) \\ - Langanhaltende Immobilität
}

\section{Prophylaxe für Personen mit höherem Risiko}

Bei Personen mit einem erhöhten Risiko muss für die risikoreiche Zeitperiode eines Langstreckenflugs grundsätzlich eine Antikoagulation in Betracht gezogen werden. In den Wochen nach einem orthopädischen Eingriff an den unteren Extremitäten ist dies besonders wichtig. Obwohl die Antikoagulation auch mit oralen Medikamenten durchgeführt werden kann, empfiehlt sich in der Regel die Injektion eines niedermolekularen Heparins kurz vor dem Flug (wie dies auch in der Schweiz üblich ist). Dabei soll dieselbe Dosis wie bei anderen Hochrisiko-Situationen verwendet werden (z.B. 5000 E Dalteparin $=$ Fragmin $^{\circledR}$ ).

Zusammengefasst von E. Gysling

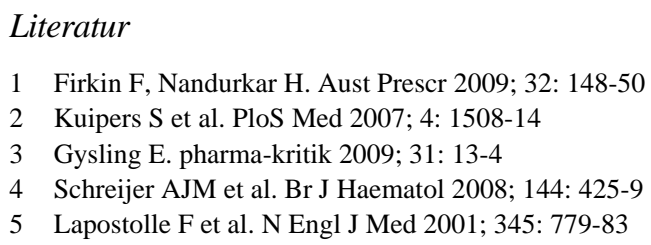

Kontrazeptiva, die nur Gestagene enthalten, haben ein geringeres Thromboembolie-Risiko. In jedem Fall dauert es nach dem Absetzen einer kombinierten Östrogen-GestagenPille etwa 2 bis 3 Monate, bis das erhöhte Risiko verschwunden ist.

\section{Prophylaxe für Personen mit geringem Risiko}

Schlaf und Hindernisse im Mittelgang verunmöglichen oft ein regelmässiges Aufstehen und Herumgehen (Aktivitäten, die grundsätzlich einer Stauung in den Beinen entgegenwirken). Gemäss einer Studie belebt nach längerem Stillsitzen eine starke Flexion der Sprunggelenke gegen einen Widerstand den venösen Rückfluss in den Beinen. Der Nutzen anderer Massnahmen, die teilweise von den Fluggesellschaften empfohlen werden (reichliche Flüssigkeitszufuhr, Bewegungsübungen, Kompressionsstrümpfe) ist bisher nicht gesichert. ${ }^{4}$ Plättchenhemmer und Antikoagulantien sind bei Personen mit einem geringen Thromboserisiko nicht indiziert.

\section{Reisende mit höherem Risiko}

Die in der Tabelle 1 genannten Faktoren sind grundsätzlich dieselben, die auch unter anderen Umständen - ohne eine Reise - ein erhöhtes Thromboserisiko darstellen. Viele Fälle treten bei Personen auf, die primär nur ein mässig erhöhtes Risiko aufweisen. ${ }^{5}$

Als hohe Risiken müssen die Vorgeschichte einer Thrombose, eine aktive Krebserkrankung und eine Herzinsuffizienz bezeichnet werden. Individuen, die eine vererbte Gerinnungsstörung (z.B. eine Faktor-V-Leiden-Mutation) aufweisen, gehören ebenfalls zu denjenigen mit erhöhtem Risiko. Anderseits ist im Hinblick auf eine Flugreise ein generelles Screening bei Gesunden, die bisher keine Thrombose gehabt haben, nicht sinnvoll. Eine entsprechende Untersuchung kann erwogen werden, wenn eine eindeutige Familienanamnese von Thrombosen vorhanden ist oder bei Frauen unter oralen Kontrazeptiva.

\author{
Fragen zur Pharmakotherapie? \\ Vielleicht können wir Ihnen helfen. Unser Informations- \\ zentrum beantwortet Ihre Fragen vertraulich, speditiv \\ und kostenlos. \\ info-pharma, Bergliweg 17, 9500 Wil \\ Telefon 071-910-0866 - Telefax 071-910-0877 \\ e-mail: sekretariat@infomed.ch \\ Ihr freiwilliger Beitrag auf Postcheckkonto 90-36-1 hilft \\ uns, diese Dienstleistung auszubauen.
}

\section{pharma-kritik}

\author{
www.pharma-kritik.ch \\ e-mail: sekretariat@infomed.ch \\ Gegründet 1979
}

von Etzel Gysling unter Mitarbeit von Renato Galeazzi und Urs A. Meyer Redaktionsteam: Renato Galeazzi, Etzel Gysling (Leitung), Urspeter Masche, Peter Ritzmann, Thomas Weissenbach

Layout und Sekretariat: Verena Gysling

Abonnementspreis für den Jahrgang 31 (2009, 20 Nummern): 102 Franken

Erscheinungsweise: 18 Ausgaben

Infomed-Verlags-AG, Bergliweg 17, $9500 \mathrm{Wil}$

Telefon 071-910-0866, Telefax 071-910-0877

Website: www.infomed.org - e-mail: sekretariat@infomed.ch

Druck: Druckerei R.-P. Zehnder AG, 9500 Wil

(C) 2010 Infomed Wil. All rights reserved. 


\section{MARKENNAMEN}

der im Text erwähnten Arzneimittel*

( $A=$ in Österreich, $D=$ in Deutschland)

\section{Chloroquin}
A: Resochin
D: Resochin

\section{Cyclopentolat}
A: Minims-Cyclopentolat
D: Zyklolat und andere

\section{Ethosuximid}
A: Petinimid, Suxinutin
D: Petmidan, Suxilep

Hydroxychloroquin
A: Plaquenil
D: Quensyl

Metronidazol
A: Anaerobex
D: Arilin, Clont, Flagyl u.a.

\section{Mexiten}
A: -
D: Tremaril

\section{Tropicamid}

A: Mydral u.a.

D: Mydriaticum Stulln, Mydrum

* Die Liste erhebt keinen Anspruch auf Vollständigkeit. Berücksichtigt wurden in erster Linie Markennamen, die von den Schweizer Namen abweichen. 